ARTICLE

\title{
Numerical Simulation of Turbulent Flow of Coolant in a Test Blanket Module of Nuclear Fusion Reactor
}

\author{
Yohji SEKI ${ }^{1, *}$, Yoichi OHNISHI ${ }^{2}$, Akira YOSHIKAWA ${ }^{1}$, Hisashi TANIGAWA ${ }^{1}$, Takanori HIROSE ${ }^{1}$, \\ Akira OHZU $^{3}$, Koichiro EZATO ${ }^{1}$, Daigo TSURU ${ }^{1}$, Satoshi SUZUKI ${ }^{1}$, Kenji YOKOYAMA ${ }^{1}$, \\ Mikio ENOEDA ${ }^{1}$, Hiroyasu TANIGAWA ${ }^{3}$ and Masatoshi KURETA ${ }^{3}$ \\ ${ }^{1}$ Japan Atomic Energy Agency, 801-1 Mukoyama, Naka-shi, Ibaraki-ken 311-0193, JAPAN \\ ${ }^{2}$ AdvanceSoft Corporation, 1-9-20 Akasaka, Minato-ku, Tokyo-to 107-0052, JAPAN \\ ${ }^{3}$ Japan Atomic Energy Agency, 2-4 Shirakata, Tokai-mura, Naka-gun, Ibaraki-ken, 319-1195, Japan
}

\begin{abstract}
Japan Atomic Energy Agency has been performing the research, development and design of a test blanket module with a water-cooled solid breeder for ITER. For our design, the TBM is mainly composed of a first wall, two side walls, a back wall and membrane panels of bulkhead sections for pebbles. The temperature of a coolant pressurized up to $15 \mathrm{MPa}$ is designed as $553 \mathrm{~K}$ and $598 \mathrm{~K}$ in an inlet and an outlet of the test blanket module, respectively.

Establishment of estimation methods of the flow phenomena is important for designs of the channel network and predictions of the material corrosion and erosion. A purpose of our research is to establish and verify the method for the prediction of the flow phenomena.

In this study, the Large-eddy simulation and Reynolds averaged Navier-Stokes simulation have been performed to predict the flow rates in the channels of the side wall. It results in the inhomogeneous flow rates at each channel. At viewpoint of the heat removal capability, however, the smallest flow-rates near the first wall are evaluated with satisfying acceptance criteria. Moreover, the results of the numerical simulation correspond with those of experiment performed for the real size mockup.
\end{abstract}

KEYWORDS: nuclear fusion reactor, test blanket module, turbulent flow, RANS, LES

\section{Introduction}

Japan Atomic Energy Agency (JAEA) has been performing the research, development and design of a test blanket module (TBM) with a water-cooled solid breeder (WCSB) for ITER as shown in Fig. 1. ${ }^{1)}$ Major design parameters are summarized in Table 1. ${ }^{2}$ Total heat deposit is $0.904 \mathrm{MW}$ and total tritium generation rate is $0.134 \mathrm{~g} / \mathrm{FPD}$. The dimension is $0.484 \mathrm{~m}(\mathrm{~W}) \times 1.66 \mathrm{~m}(\mathrm{H}) \times 0.6 \mathrm{~m}(\mathrm{~T})$.

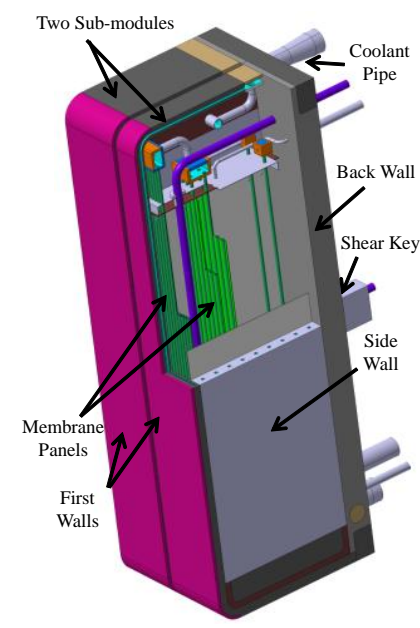

Fig. 1 Structure of the WCSB TBM

*Corresponding author, E-mail: seki.yohji@jaea.go.jp
For our design, two sub-modules constitute one TBM. The sub-module of the TBM is mainly composed of a first wall, two side walls, a common back wall and multiple membrane panels of bulkhead sections for pebbles. The membrane panels are heated by heated pebbles of solid tritium breeders and neutron multipliers. The first wall is heated by an averaged surface heat flux of $0.3 \mathrm{MW} / \mathrm{m}^{2}$. Moreover, all of the structural materials are heated by a neutron wall loading of $0.78 \mathrm{MW} / \mathrm{m}^{2}$ from the plasma.

Table 1 Specification of the WCSB TBM

\begin{tabular}{ccc}
\hline Items & Unit & Material and value \\
\hline Structural Material & & $\mathrm{F} 82 \mathrm{H}$ \\
Coolant & & Water \\
Multiplier & & $\mathrm{Be}, \mathrm{BeTi}$ alloy \\
Breeder & & $\mathrm{Li}_{2} \mathrm{TiO}_{3}$, \\
& & other Li ceramics \\
Area of First Wall & $\mathrm{m}^{2}$ & $0.484 \times 1.66$ \\
TBM Thickness & $\mathrm{m}$ & 0.6 \\
Surface Heat Flux (max.) & $\mathrm{MW} / \mathrm{m}^{2}$ & $0.3(0.5)$ \\
Nuetron Wall Load & $\mathrm{MW} / \mathrm{m}^{2}$ & 0.78 \\
Total Heat Deposit & $\mathrm{MW}$ & 0.904 \\
Total Tritium Production & $\mathrm{g} / \mathrm{FPD}$ & 0.134 \\
Coolant Pressure & $\mathrm{MPa}$ & 15.5 \\
Coolant Inlet Temperature & ${ }^{\circ} \mathrm{C}$ & 280.0 \\
Coolant Outlet Temperature & ${ }^{\circ} \mathrm{C}$ & 325.0 \\
Coolant Flow Rate per sub-module & $\mathrm{kg} / \mathrm{s}$ & 3.0 \\
\hline
\end{tabular}




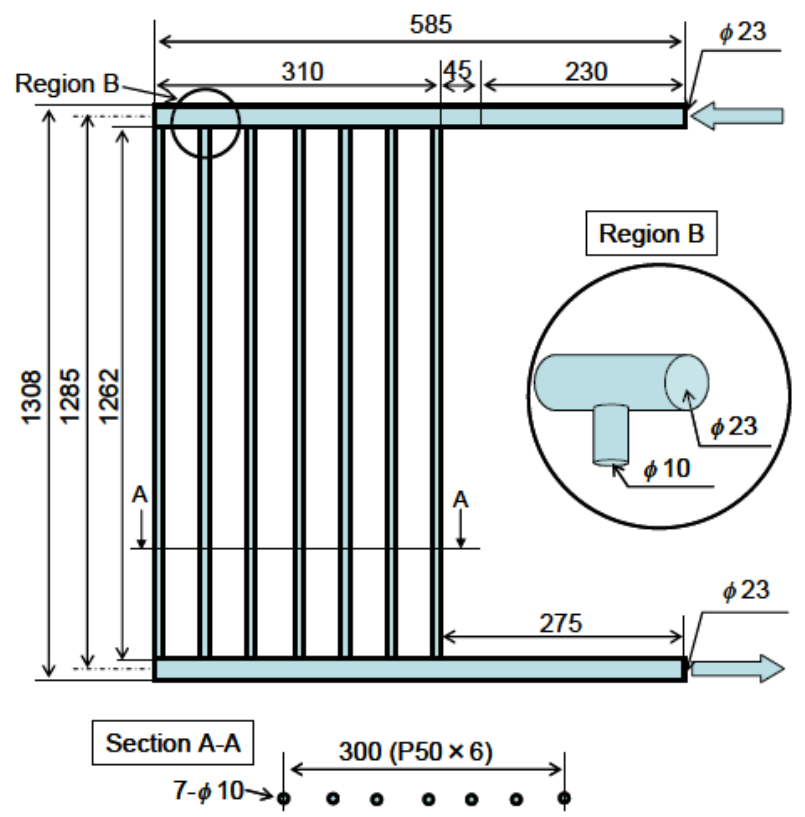

Fig. 2 Coolant channels of side wall in millimeters

Therefore, the components of the TBM include a lot of cooling channels. The temperature of a coolant pressurized up to $15 \mathrm{MPa}$ is designed as $553 \mathrm{~K}$ and $598 \mathrm{~K}$ in an inlet and an outlet of the TBM, respectively. In the case of the side wall, the circular channel is applied as the channel geometries as seen in Fig. 2. In the case of a real size mockup, both the two manifolds (diameter of $23 \mathrm{~mm}$ ) and the seven branch channels (diameter of $10 \mathrm{~mm}$ ) are bored into the side wall (board thickness of $30 \mathrm{~mm}$ ) by drilling. It has the advantage that the breeder region can increase by housing the channels into the side wall. However, it is expected that the flow rates differ from one branch channel to another. It remains a risk that a significant problem of decrease in a heat removal capability takes place.

Establishment of estimation methods of the flow phenomena is important for designs of the channel network and predictions of the material corrosion and erosion. Although the theoretical analysis and experiment as methods of predictions have been in use as powerful techniques so far, in recent years, the computational analysis has become acknowledged the important prediction technique of turbulent flows. A purpose of our research is to establish and verify the method for the prediction of the flow phenomena.

In this study, the Large-eddy simulation (LES) and Reynolds averaged Navier-Stokes simulation (RANS) have been performed to predict the flow rates in the channels of the side wall. It results in the inhomogeneous flow rates at each channel. At viewpoint of the heat removal capability, however, the smallest flow-rates near the first wall are evaluated with satisfying acceptance criteria. Moreover, the results of the numerical simulation correspond with those of experiment performed for the real size mockup.

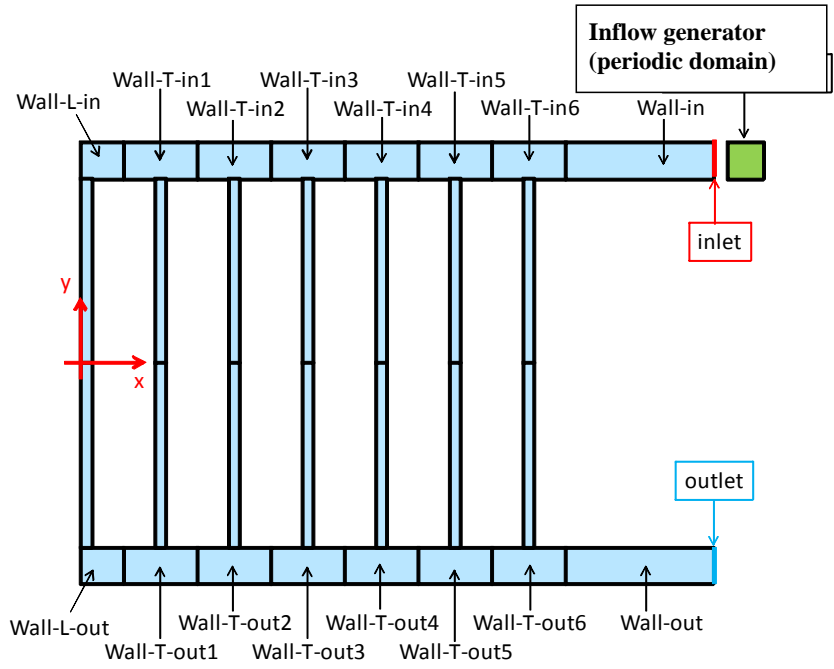

Fig. 3 Schematic diagram of the flow region and labels in the side wall

\section{Numerical Procedures}

\section{Computational Domain and Condition}

The configuration of the numerical simulation is constituted by seven channels and two headers as shown in Fig. 3. The computational domain is separated and labeled to improve understanding.

Computational condition is summarized in Table 2. In the case 1 , the pressurized water at high temperature is applied to simulate the coolant condition in the side wall of the WCSB TBM. On the other hand, in the case 2, water at low temperature and pressure are applied to compare with the result of experiment which was performed at the room temperature. $^{3)}$ In the present numerical simulation, the temperatures of fluid in the Case 1 and 2 are uniformly set to be $300{ }^{\circ} \mathrm{C}$ and $19.5^{\circ} \mathrm{C}$, respectively. Adiabatic wall is supposed to thermal boundary condition. Properties of water for the temperature are accordance with JSME Steam tables. ${ }^{3)}$ Euler's implicit method both cases is applied to temporal progress.

\section{(1) LES}

As for the LES, the Navier-Stokes equation for an unsteady incompressible viscous flow is solved using the standard Smagorinsky model. Smagorinsky constant is set to be $0.1{ }^{4)}$ Figure 3 shows the inflow generator upstream from

Table 2 Computational condition

\begin{tabular}{ccc}
\hline Fluid condition & Case 1 & Case 2 \\
\hline Temperature $\left({ }^{\circ} \mathrm{C}\right)$ & 300.0 & 19.5 \\
Pressure $(\mathrm{MPa})$ & 15.0 & 0.3 \\
Inlet Flow rate $(\mathrm{kg} / \mathrm{s})$ & 1.5 & 1.0 \\
Wall boundary & Non-slip & Non-slip \\
Computational method & LES & RNS \\
Grid-number (Mega) & Around 340 & Around 290 \\
Cpu-number & 128 & 128 \\
Discretization scheme & 2nd order central and & 1st upwind \\
& 1st upwind & and 2nd upwind \\
\hline
\end{tabular}


the inlet. The turbulent flow at the inlet is often generated at a separate computational domain in which the flow is fully developed by a spatially periodic boundary condition so that the realistic inflow is able to be approximated as the fully developed turbulent flow. In the present LES, a fully developed turbulent pipe flow is generated as an inflow condition at each time step by using the inflow generator. An axial dimension of $L / D=1$ is set to be subject to a streamwise periodic boundary condition as schematically shown in Fig. 3.

Moreover, first calculation is performed by the RANS until an initial field becomes fully developed turbulent flow before beginning collection of a statistical data. After confirming steady state of averaged values such as the pressure and the velocity at whole computational domain, the turbulence statistics were started obtaining.

(2) RANS

In the case of RANS, the Navier-Stokes equation for a steady incompressible viscous flow is solved using the Re-normalization group (RNG) method. ${ }^{5)}$

The fully developed turbulent pipe flow is prepared by a preliminary calculation to use as inflow condition. The same profile of the flow is applied to the inlet flow at each time step.

An enhanced wall treatment is applied to RANS. ${ }^{6)}$ Moreover, spatial resolutions near the wall especially at the elbow and $\mathrm{T}$-junction are smaller than $\mathrm{y}^{+}=1.0$, where the superscript (+) indicates normalization by a kinematic viscosity coefficient and a friction velocity. At the region of the separation and reattachment of the flow near the wall, the type of "low-Reynolds-number" is selected as the turbulence model. This is because a distribution of flow near the wall is of importance to accurately evaluate the wall shear stress.

\section{Results and Discussion}

\section{Wall Shear Stress}

Figure 4 shows a time-averaged wall shear stress of case 1 at the region labeled "Wall-T-in6" in Fig. 3. In this study, large values of the wall shear stress clearly and locally appear at edge of $\mathrm{T}$-junction. The $\mathrm{T}$-junction is divided to three regions which exhibit $700 \mathrm{~Pa}, 500 \mathrm{~Pa}$ and $100 \mathrm{~Pa}$ in Fig. 4. The time-averaged velocity fields in the case 1 are shown in Fig. 5. The contour on the wall indicates the time-averaged wall shear stress which corresponds to that of Fig. 4. Moreover, a line indicates a path line which corresponds to a streamline because of the steady statistic. The color of the line shows the magnitude of the velocity. These figures indicate that the coolant water with a swirl structure flows from the main pipe to the branch pipe. Therefore, the distribution of shear stress is also caused by swirl structure flows.

\section{The Comparison with an Experimental Result}

As for the design of the structure of the coolant channels in the side wall, an experimental study is also performed. ${ }^{7)}$ In the real sized side wall, the required water flow rate was estimated so that the side wall can be kept under allowable temperature. Using the manufactured mockup, the water flow experiment was conducted, and the water flow rates in the branch channels were measured by an ultrasonic flow-

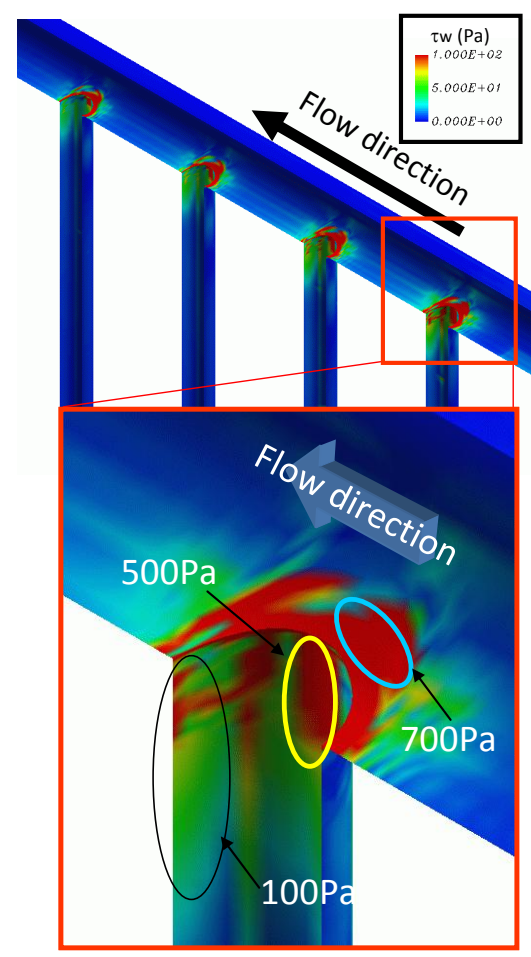

Fig. 4 Time-averaged wall shear stress $\left(\tau_{\mathrm{w}}\right)$ in the case 1

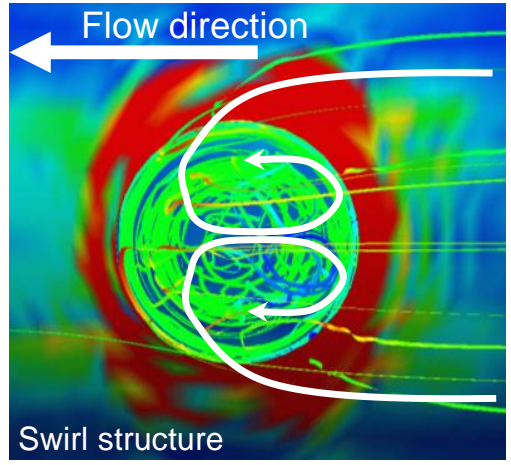

(a) Top view

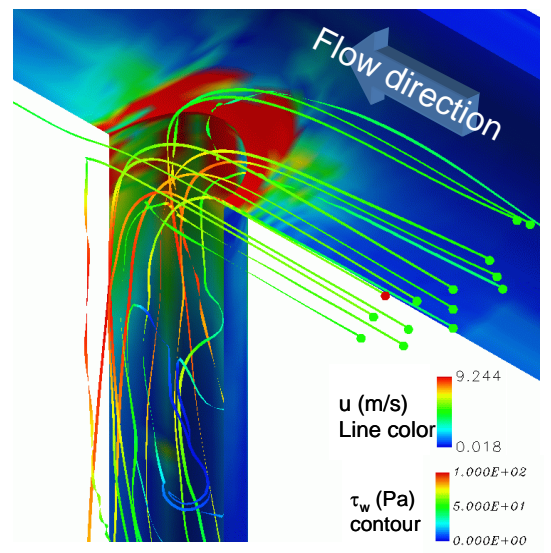

(b) Bird's eye

Fig. 5 Time-averaged velocity in the case 1

meter. The flow rates obtained by the RANS of case 2 are compared with the measured those to evaluate the accuracy of prediction.

Table 3 shows the comparison of flow rate between ex- 
Table 3 Comparison of flow rate between experiment and RANS (Case 2)

\begin{tabular}{cccc}
\hline Position & RANS $(\mathrm{kg} / \mathrm{s})$ & Exp. $(\mathrm{kg} / \mathrm{s})$ & Margin $(\%)$ \\
\hline Wall-T-6 & 0.153 & 0.157 & 2.6 \\
Wall-T-5 & 0.149 & 0.142 & -4.7 \\
Wall-T-4 & 0.145 & 0.151 & 4.1 \\
Wall-T-3 & 0.142 & 0.139 & -2.1 \\
Wall-T-2 & 0.139 & 0.151 & 8.6 \\
Wall-T-1 & 0.137 & 0.134 & -2.2 \\
Wall-L & 0.133 & 0.125 & -6.0 \\
\hline
\end{tabular}

perimental result and RANS one of Case 2 after 1.0 second. These flow rates obtained by RANS are in good agreement with experimental results. It indicates that RANS was verified to available method of prediction for design of the multiple coolant channels.

In the case 1 by using LES, the purpose was not only to recognize flow phenomenon but also to predict the flow distribution at high temperature condition of coolant. At the next step, LES will be performed at the coolant condition of the room temperature to elaborate the LES is able to predict the flow-distribution at each channel at any temperature condition.

\section{Conclusion}

In this study, the Large-eddy simulation and Reynolds averaged Navier-Stokes simulation have been performed to predict the flow rates in the channels of the side wall. Evaluation of wall shear stress is performed with based on the flow distribution. It results in the inhomogeneous flow rates at each channel. The results of the numerical simulation correspond with those of experiment performed for the real size mockup.

\section{Acknowledgment}

The "FrontFlow/red" computer program was created by the members of the national project "Frontier Simulation Software for Industrial Science (FSIS)", and AdvanceSoft Corporation has developed and released this software as "Advance/FrontFlow/red" (http://www.advancesoft.jp/).

\section{References}

1) M. Enoeda, M. Akiba, S. Tanaka, A. Shimizu, A. Hasegawa, S. Konishi, A. Kimura, A. Kohyama, A. Sagara, T. Muroga, "Overview of design and R\&D of test blankets in Japan," Fusion Eng. Des., 81, 415-424 (2006).

2) Y. Nomoto, S. Suzuki, K. Ezato, T. Hirose, D. Tsuru, H. Tanigawa, T. Hatano, M. Enoeda, M. Akiba, "Structural concept of Japanese solid breeder test blanket modules for ITER," Fusion Eng. Des., 81, 719-724 (2006).

3) Japan Society of Mechanical Engineers, JSME Steam Tables (1999).

4) A. Yoshizawa, S. Murakami, T. Kobayashi, N. Taniguchi, Y. Dai, A. Kuroda, K. Kamemoto, S. Kato, Y. Nagano, T. Tsuji, "Analysis of Turbulent Flows, computational fluid dynamics series 3," University of Tokyo Press, 42[3], 67-118 (2003), [in Japanese].

5) V. Yakhot, S. A. Orszag, "Renormalization group analysis of turbulence. I.Basic theory," J. Sci. Comput., 1, 3-51 (1986).

6) H. C. Chen, V. C. Patel, "Near-Wall turbulence models for complex flows including separation," AIAA J., 26, 641-648 (1988)

7) A. Yoshikawa, H. Tanigawa, Y. Seki, T. Hirose, D. Tsuru, K. Ezato, Y. Yokoyama, S. Suzuki, M. Enoeda, "Non-uniform Water Flow Distribution in Side Wall for Japanese Test Blanket Module," J. Nucl. Sci. Technol., to be appeared (2012). 